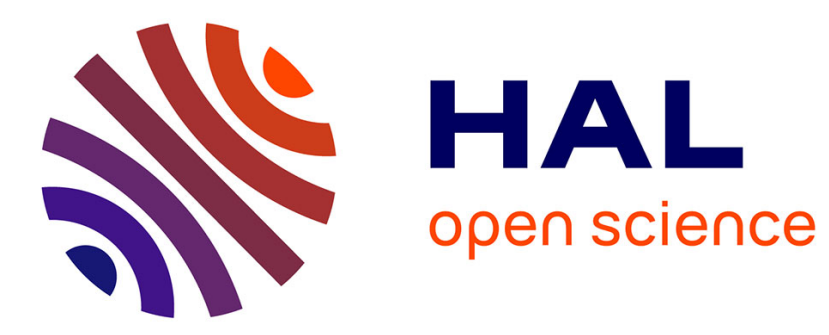

\title{
Identification and Validation of a Non Symmetrical System Level EMC Model for Power Electronics Converter
}

Blazej Czerniewski, J-L. Schanen, Herve Chazal, Pericle Zanchetta, Caio Fonseca de Freitas

\section{To cite this version:}

Blazej Czerniewski, J-L. Schanen, Herve Chazal, Pericle Zanchetta, Caio Fonseca de Freitas. Identification and Validation of a Non Symmetrical System Level EMC Model for Power Electronics Converter. 2021 IEEE Energy Conversion Congress and Exposition (ECCE2021), Apr 2021, Vancouver (virtual), Canada. 10.1109/ECCE47101.2021.9595780 . hal-03434094

\author{
HAL Id: hal-03434094 \\ https://hal.science/hal-03434094
}

Submitted on 18 Nov 2021

HAL is a multi-disciplinary open access archive for the deposit and dissemination of scientific research documents, whether they are published or not. The documents may come from teaching and research institutions in France or abroad, or from public or private research centers.
L'archive ouverte pluridisciplinaire $\mathbf{H A L}$, est destinée au dépôt et à la diffusion de documents scientifiques de niveau recherche, publiés ou non, émanant des établissements d'enseignement et de recherche français ou étrangers, des laboratoires publics ou privés. 


\section{Identification and Validation of a Non Symmetrical System Level EMC Model for Power Electronics Converter}

\author{
Blazej Czerniewski \\ Univ Grenoble Alpes G2ELab \\ Grenoble, France \\ and Dept of Electrical and Electronic \\ Eng. University of Nottingham, UK \\ blazej.czerniewski@gmail.com \\ Pericle Zanchetta \\ Dept of Electrical and Electronic Eng. \\ University of Nottingham, UK \\ and Dept of Electrical, Computer and \\ Biomedical Eng. \\ University of Pavia, Italy \\ Pericle.Zanchetta@nottingham.ac.uk
}

\author{
Jean-Luc Schanen \\ Univ Grenoble Alpes G2ELab \\ Grenoble, France \\ jean-luc.schanen@g2elab.grenoble- \\ inp.fr
}

\author{
Herve Chazal \\ Univ Grenoble Alpes G2ELab \\ Grenoble, France \\ herve.chazal@g2elab.grenoble-inp.fr
}

\begin{abstract}
This paper deals with system level EMC models for power electronics converter, able to investigate the conducted emissions in embedded grid, for instance. The model topology presented in this work is not symmetric and its identification does not rely on the usual Differential / Common Mode separation. The identification method is presented, and the model is validated in different asymmetric environments: a specific configuration with a modified LISN, and another one with minus connected to ground. Both approaches are carried out in simulation and experiment, for the simple example of a boost converter.
\end{abstract}

Keywords-Electromagnetic Electromagnetic Interference, Identification, Power Electronics.

\section{INTRODUCTION}

Compatibility, EMC, EMI, Embedded Grid,

Since Power Electronics appeared in the $70 \mathrm{~s}$, lot of improvements have been made on semiconductor technology, leading to an incredible interest for this energy conversion process: so easy to use, so high efficiency and so various conversion capability have spread power electronics almost everywhere. The only remaining issue is the switching process itself, which generates huge transients and therefore so-called electromagnetic interferences (EMI). The need of EMI mitigation has been standardized in the $90 \mathrm{~s}$, and is still evolving with the new high-speed devices coming on the market. The characterization of a converter in a standardized environment, connecting it on a Line Impedance Stabilization Network (LISN), allows reproducibility of the measurement, but does not really correspond to a real case, where many converters are connected to a same grid. Therefore, for complex systems including several converters and cabling, a system level model should be found in order to conduct a real investigation of the EMC disturbances generated in this system, which will not simply result from the individual sum of each single converter. Indeed, the grid impedance may be significantly different from the LISN one, and interactions between converters may arise [1]. Furthermore, to keep confidentiality on the internal description of converters, black box models are really interesting to be used [2]. Up to now, the majority of the models developed in this approach use the

This work was funded by DGA-DGAC project MECEP: Mastering Conducted Emissions on Power Equipment. N²016 930800 classical common mode differential mode decomposition [2], and are intrinsically symmetric [2][3][4]. They also have been validated in symmetrical conditions. In this paper an identification method of an asymmetrical model is proposed, what prevents using the conventional mode separation for identification. The model is then validated in both simulation and measurements, in the simple example of a boost converter used in two different test cases. Section II will present the model and the identification method as well as the impact of asymmetry on the mode separation. Two asymmetrical test cases will also be introduced to allow model validation. Section III will apply the model to the asymmetrical test cases in simulation, and Section IV will deal with experimental validation. Section V will contain discussion and conclusion.

\section{MODEL AND IDENTIFICATION METHOD}

\section{A. Model topology}

In the "black box" representation [2][3][4] the converter is seen from its external connections. Several topologies of this terminal model have been proposed, among them Norton based [4] or hybrid (Fig. 1 [2]). The identification of the model parameters was reported using two different approaches. The first one uses line current measurements when the converter is operating on known impedances. Changing these impedances allows identifying the model parameters. A detailed report on this identification approach is proposed in [3]. Unterminated models have also been proposed for investigating EMI on both source and load sides [5], what is especially interesting when load is changing, or in the case of a converter interconnecting two different grids.

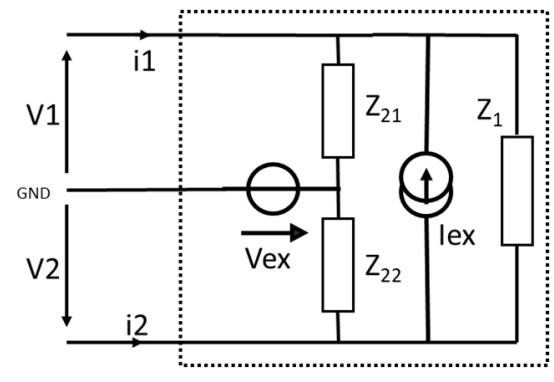

converter

Fig. 1. "Black Box" EMC Model, using an hybrid representation with one current source and one voltage source. 
A second method [2] uses off line impedance measurements and combines them with line current measurement on a known environment, e.g. a LISN, to obtain the two sources, Vex and Iex. Vex is supposed to be the source of Common Mode (CM), Iex the source of Differential Mode (DM). This method uses the assumption of decoupled CM and $\mathrm{DM}$, and therefore supposes the converter symmetrical, as it will be explained in subsection B. Therefore the impedances $Z_{21}$ and $Z_{22}$ in Fig. 1 are assumed identical (named $Z_{2}$ ). The first method from [3] uses the same assumption. In both [2] and [3], the model being symmetric, it has been validated in symmetrical conditions only. Model from [6] has investigated unbalanced situations, but starting from the converter topology. In this paper, the model identification is supposed to use external measurements only, without any a priori knowledge on the converter layout. Subsection B starts by studying the impact of an asymmetrical model and an asymmetrical grid on the Common and Differential mode separation. The identification method and equations for $\mathrm{Z}_{21} \neq \mathrm{Z}_{22}$ are provided in subsection $\mathrm{C}$. Subsection $\mathrm{D}$ introduces asymmetrical test conditions, to validate the model.

\section{B. Symetry and Mode Separation}

The model of Fig. 1 is connected to a grid, represented with three impedances $Z_{\mathrm{G} 1}, Z_{\mathrm{G} 21}$ and $\mathrm{Z}_{\mathrm{G} 22}$ Fig. 2. It is worth linking Differential Mode current $I_{D M}=\frac{1}{2} \cdot\left(I_{1}-I_{2}\right)$ and Common Mode current $I_{C M}=I_{1}+I_{2}$ with the two excitation sources Vex and Iex. In the general case, it is quite obvious that $I_{1}$ and $I_{2}$ depend on both Vex and Iex, and of course the same for $\mathrm{I}_{\mathrm{DM}}$ and $\mathrm{I}_{\mathrm{CM}}$. In other words, Common Mode and Differential Mode currents depends on both voltage and current sources of the model, Vex and Iex. However, if we consider symmetric model and grids, i.e. $Z_{21}=Z_{22}=Z_{2}$ and $\mathrm{Z}_{\mathrm{G} 21}=\mathrm{Z}_{\mathrm{G} 22}=\mathrm{Z}_{\mathrm{G} 2}$, the quite complex expressions of $\mathrm{DM}$ and $\mathrm{CM}$ currents become more simple:

$$
\begin{gathered}
I_{D M}=\frac{z_{1} \cdot Z_{2} \cdot\left(Z_{G 1}+2 \cdot z_{G 2}\right)}{Z_{1} \cdot Z_{2} \cdot Z_{G 1}+2 \cdot Z_{1} \cdot Z_{2} \cdot z_{G 2}+Z_{1} \cdot Z_{G 1} \cdot z_{G 2}+2 \cdot Z_{2} \cdot Z_{G 1} \cdot z_{G 2}} \cdot \text { Iex } \\
I_{C M}=\frac{2}{Z_{2}+Z_{G 2}} \cdot \text { Vex }
\end{gathered}
$$

Eq (1) is simply the current divider of Iex between $Z_{1} / /\left(2 \cdot Z_{2}\right)$ and $Z_{G 1} / /\left(2 \cdot Z_{G 2}\right)$, and does not depend on Vex. Eq (2) means that the common mode current is equal to Vex divided by the impedance $\left(Z_{2}+Z_{\mathrm{G} 2}\right) / 2$, since the two branches $\left(Z_{2}+Z_{\mathrm{G} 2}\right)$ are in parallel, and no current is circulating in $Z_{1}$ and $Z_{\mathrm{G} 1}$, due to symmetry.

Therefore, the usual model needs symmetry of both the model and the grid, to link CM current to Vex only, and DM current to Iex only. However, this situation is not common in the general case, and both sources of the model contribute to $\mathrm{CM}$ and DM generation. Consequently, identifying a symmetrical model, even with symmetric grid, with the assumption of mode separation as in [2] may be wrong. Also using a symmetrical model in an asymmetrical grid could lead to wrong results. That's why the following section proposes to identify an asymmetrical model, with $Z_{21} \neq Z_{22}$.

\section{Asymmetrical Model Identification}

The assumption of "masking impedance" used in [3] and also demonstrated in [7], allows justifying the use of constant impedance whatever the semiconductors state. It means that the impedance seen from converter terminals is not modified significantly when the switches change state. Therefore, the method proposed in [2] measures the off line impedances of the converter in Differential Mode and Common Mode configurations using an impedance measurement device.

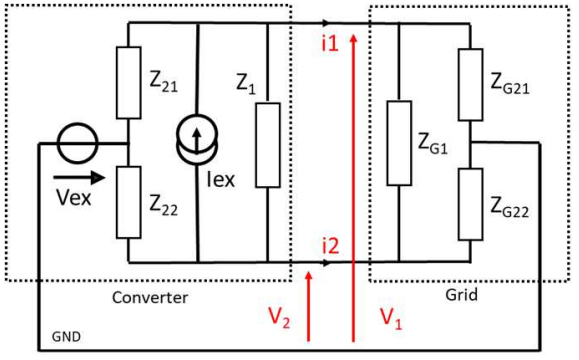

Fig. 2. Connection of the black box model to a grid, represented by 3 impedances $\mathrm{Z}_{\mathrm{G} 1}, \mathrm{Z}_{\mathrm{G} 21}, \mathrm{Z}_{\mathrm{G} 22}$.

In this paper, to identify three different impedances $Z_{1}, Z_{21}$, and $Z_{22}$, the three different configurations of Fig. 3 are used, corresponding to following equations:

$$
\begin{aligned}
Z_{1} & =\frac{2 \cdot Z_{M G} \cdot Z_{P G} \cdot Z_{P M}}{Z_{M G} \cdot Z_{P M}-Z_{M G} \cdot Z_{P G}+Z_{P G} \cdot Z_{P M}} \\
Z_{21} & =\frac{2 \cdot Z_{M G} \cdot Z_{P G} \cdot Z_{P M}}{Z_{M G} \cdot Z_{P G}-Z_{M G} \cdot Z_{P M}+Z_{P G} \cdot Z_{P M}} \\
Z_{22} & =\frac{2 \cdot Z_{M G} \cdot Z_{P G} \cdot Z_{P M}}{Z_{M G} \cdot Z_{P G}+Z_{M G} \cdot Z_{P M}-Z_{P G} \cdot Z_{P M}}
\end{aligned}
$$

These measurement configurations are more precise than in [2], since no "floating" measurements are needed (Plus to Minus impedance). The minus of the measurement setup ("cold" point) is always connected to the ground of the converter. This allow reducing the amount of CM current derived to the ground (common mode "leakage"), and keeping plus and minus currents almost identical, what is necessary to identify an impedance $\mathrm{V}_{\mathrm{OSC}} / \mathrm{I}_{\text {meas. }}$. Fig. 4 illustrates the effect of CM leakage, which can be avoided using a BalUN (Balanced-Unbalanced Network [8]). However this complicates measurements, due to more difficult calibration.

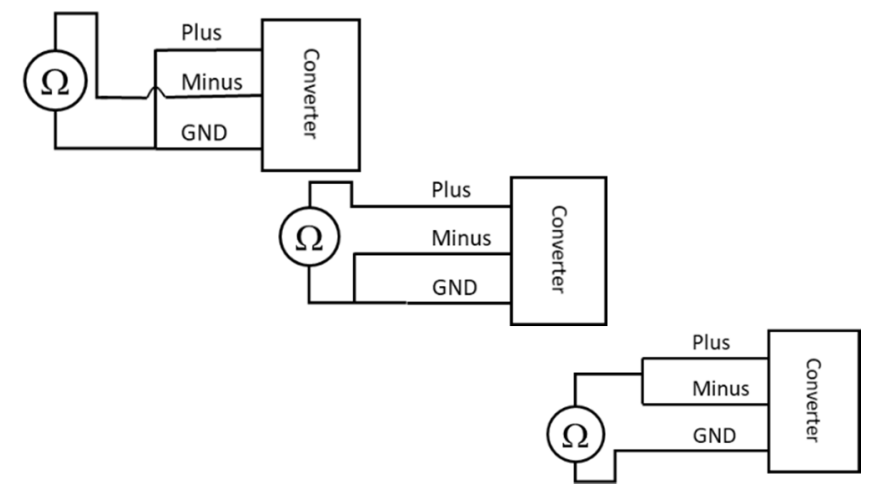

Fig. 3. The 3 impedance measurement configurations to identify the three impedances of the model. Top: $Z_{\mathrm{PG}}$ with Plus and Ground connected, Middle: $Z_{\mathrm{MG}}$ with Minus and Ground connected, Bottom: $Z_{\mathrm{PM}}$, with Plus and Minus connected.
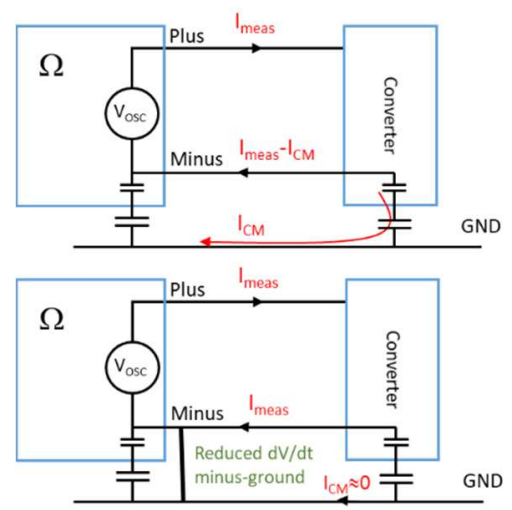

Fig. 4. Reducing potential $\mathrm{CM}$ circulation when connecting the impedance bridge to the ground. 
Once the impedances $Z_{1}, Z_{21}$, and $Z_{22}$ have been identified, the two remaining unknowns are the two sources Vex and Iex, as a function o frequency. A simple measurement of the line currents in a known situation (for instance converter connected to a LISN) allows the determination of Iex and Vex. Eq. (6) and (7) provide the expressions of Iex and Vex as a function of measured line currents $I_{1}$ and $I_{2}, Z_{L}$ being the line to ground impedance of the LISN. The electrical circuit of the considered LISN is displayed in Fig. 5, associated values are provided in TABLE I. It is worth noting that these currents must be recorded together to keep the information of the phase shift. Indeed the computation performed in Eq. (6) and Eq. (7) are using complex impedances. A spectrum analyzer cannot be used since the phase shift is not available. Time domain waveforms + FFT must therefore be used. A lot of care has to been taken for a proper recording of the experimental waveforms (section IV). $50 \mathrm{MHz}$ passive current probes were used, signals were captured using a high amount of data points (500k) and a high dynamic range (11bits digital scope [9]).

$$
\begin{gathered}
V_{e x}=I_{1} \cdot \frac{Z_{L} \cdot Z_{22}+Z_{21} \cdot Z_{22}}{Z_{21}+Z_{22}}+I_{2} \frac{Z_{L} \cdot Z_{21}+Z_{21} \cdot Z_{22}}{Z_{21}+Z_{22}} \\
I_{e x}=-I_{1} \frac{Z_{1} \cdot Z_{L}+Z_{1} \cdot Z_{21}+Z_{L} \cdot Z_{21}+Z_{L} \cdot Z_{22}}{Z_{1} \cdot Z_{21}+Z_{1} \cdot Z_{22}}+I_{2} . \\
\frac{Z_{1} \cdot Z_{L}+Z_{1} \cdot Z_{22}+Z_{L} \cdot Z_{21}+Z_{L} \cdot Z_{22}}{Z_{1} \cdot Z_{21}+Z_{1} \cdot Z_{22}}
\end{gathered}
$$

TABLE I. LISN PARAMETERS

\begin{tabular}{|l|l|l|l|}
\hline $\mathrm{LN}[\mu \mathrm{H}]$ & 250 & $\mathrm{R}[\Omega]$ & 50 \\
\hline $\mathrm{CN}[\mathrm{nF}]$ & 220 & $r[\Omega]$ & 5 \\
\hline $\mathrm{Cr}[\mu \mathrm{F}]$ & 1 & $l[\mu \mathrm{H}]$ & 50 \\
\hline
\end{tabular}

Fig. 5. Line Impedance Stabilization Network (symmetrical) used for converter identification.

\section{Test Configurations}

To validate the identified model (Vex, Iex, $Z_{1}, Z_{21}$, and $Z_{22}$ ), two asymmetrical test configurations have been chosen, since symmetrical model was already confirmed for symmetrical cases in previous works [2][3][4]:

- $\quad$ A modified LISN with minus to ground impedance $\mathrm{R}$ being equal to $5 \Omega$ instead of $50 \Omega$, the plus to ground remaining $50 \Omega$.

- A minus to ground connection on the converter side, displayed in Fig. 6 (the impedance of the link is not exactly zero, as explained later).

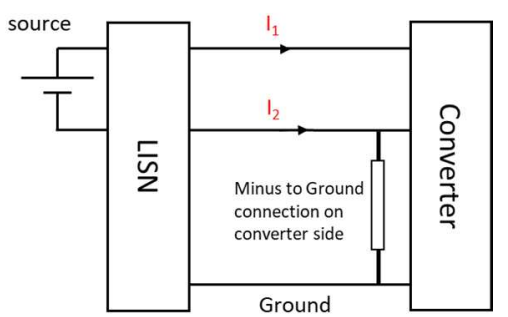

Fig. 6. Validation case "Minus to ground". The minus of the converter is connected to the ground through an almost zero impedance (actually $5 \mathrm{nH}-$ $265 \mathrm{~m} \Omega$ ). The LISN minus is not connected to the ground.
The asymmetric LISN does not correspond to any EMC standard, it is just to provide a non symmetrical grid. This situation is not uncommon in embedded grids (minus or neutral connected to chassis for instance, directly or through an impedance).

\section{SimULATION RESULTS}

First, a simulation-based approach has been carried out. The converter is a simple boost, operating at $115 \mathrm{kHz}$ with a $2 / 3$ duty cycle. Input voltage is $14 \mathrm{~V}$ and input current $3 \mathrm{~A}$. Output voltage is $42 \mathrm{~V}$. Fig. 7 shows the equivalent circuit of the converter, including all stray elements, which have been identified from the actual converter studied in section IV. TABLE II. summaries all values of the electrical circuit. The stray inductances $\mathrm{Lp}$ and $\mathrm{Lm}$ represent the contribution of the wires between the input connectors of the converter and the input capacitor Cin. Classical Esl-Esr circuits are used to represent the electrolytic capacitors Cin and Cout. Cout is paralleled with a decoupling capacitor Cdec, supposed to be ideal. Input inductor L is modeled with Epc and Epr (equivalent parallel capacitance and resistance). Three stray capacitances accounts for the PCB tracks behavior with respect to the ground: $\mathrm{Cpg}$ between plus and ground, $\mathrm{Cmg}$ between minus and ground, and $\mathrm{Cpm}$ between the middle point of the switching leg and the ground. This latter capacitance is originating most part of common mode current.

The identification of the off line impedance is carried out in simulation using a simple AC Sweep analysis, and impedances of the model are extracted using equations (3)(4)-(5). A time simulation is then run, the converter being connected to the symmetric LISN of Fig. 5. A FFT analysis of line currents $I_{1}$ and $I_{2}$ (modulus and phase shift) allows obtaining Vex and Iex as a function of frequency, using equations (6) and (7).

Once identified in simulation, the model is used in the two test configurations detailed in subsection II-D. A specific tool has been built to solve the circuit equations in the frequency domain, in order to obtain the line currents $I_{1}$ and $I_{2}$, measured between the converter and the LISN (Fig. 6). These results are compared with a time simulation + FFT in the same configuration. It is worth noting that this is an actual validation of the model, since this one has been identified on a symmetric LISN, and is now used in a different configuration: either asymmetric LISN, or minus to ground connection on the converter side.

\section{TABLE II. BOOST MOdEl PARAMETERS}

\begin{tabular}{|l|l|l|l|l|l|}
\hline $\mathrm{Lp}[\mathrm{nH}]$ & 140 & $\mathrm{~L}[\mu \mathrm{H}]$ & 18.5 & $\mathrm{Cpg}[\mathrm{pF}]$ & 300 \\
\hline $\mathrm{Lm}[\mathrm{nH}]$ & 140 & $\operatorname{Epr}[\mathrm{k} \Omega]$ & 3.96 & $\mathrm{Cmg}[\mathrm{pF}]$ & 300 \\
\hline Cin $[\mathrm{mF}]$ & 4.7 & $\operatorname{Epc}[\mathrm{pF}]$ & 13 & $\mathrm{Cpm}[\mathrm{pF}]$ & 240 \\
\hline Esl_Cin $[\mathrm{nH}]$ & 40 & Cout $[\mu \mathrm{F}]$ & 470 & $\mathrm{Cdec}[\mu \mathrm{F}]$ & 1 \\
\hline Esr_Cin $[\mathrm{m} \Omega]$ & 60 & Esl_Cout $[\mathrm{nH}]$ & 40 & Rload & 42 \\
\hline & & Esr_Cout $[\mathrm{m} \Omega]$ & 60 & & \\
\hline
\end{tabular}

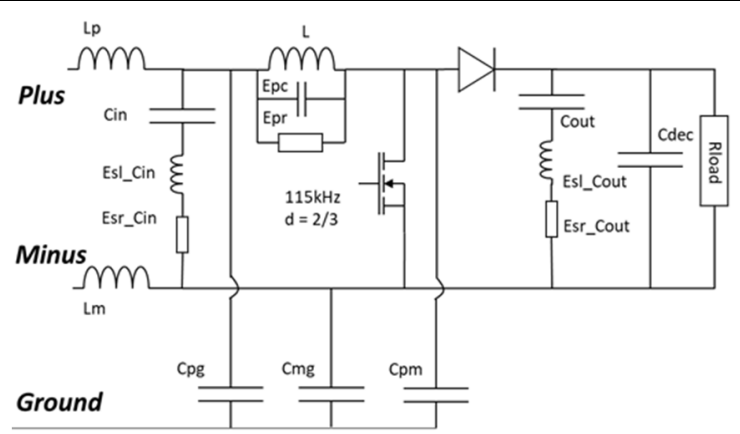

Fig. 7. Equivalent circuit of the Boost converter used for simulation. 

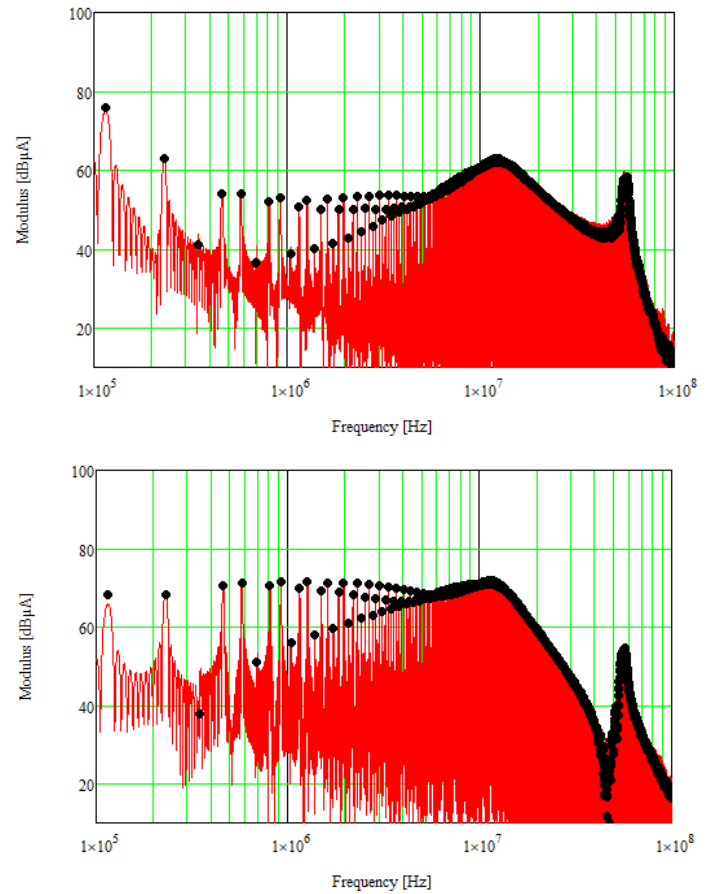

Fig. 8. Comparison between frequency model (black points) and time domain simulation + FFT (red) for asymmetric LISN (Top $\mathrm{I}_{1}$, Bottom $\mathrm{I}_{2}$ )

Fig. 8 displays the results of the line currents $\mathrm{I}_{1}$ and $\mathrm{I}_{2}$ (in $\mathrm{dB} \mu \mathrm{A}$ ) for the case of asymmetric LISN. It can be noticed that $I_{2}$ has a higher amplitude as $I_{1}$, for harmonics higher than the fundamental. This is due to lower line to ground impedance, and therefore larger contribution of CM current, which is no more balanced in each line, due to asymmetry of the grid. The phenomenon is well captured by the model on the whole frequency range of interest $(100 \mathrm{kHz}-100 \mathrm{MHz})$. The two resonances, one at $11 \mathrm{MHz}$, the other at $55 \mathrm{MHz}$ are well reproduced also. Despite the model is used in another configuration than the one used for identification, the comparison is very good. This validates the assumption of the "masking impedance" concept, which allow using impedances measured when the converter is in the off state, combined with voltage and current sources, to represent an intrinsically switching behavior. Of course, this validation has only been checked in the specific case of the studied boost converter, and is not general, but it is a promising result for this kind of "Black Box" modeling approach.

Fig. 9 show the comparison between the model and the results obtained from the time domain simulation for the "minus to ground" test case. The results are again very good, what validates further the model, even in this extreme case. It is worth noting that $\mathrm{I} 2$ current becomes very low $(30 \mathrm{~dB}$ less than $I_{1}$ ), since all current goes through the ground, and not through the minus wire. However the link between converter minus and ground being not perfect $(5 \mathrm{nH}-265 \mathrm{~m} \Omega)$, the line current $\mathrm{I}_{2}$ is not exactly zero.

The interest of this validation in simulation is that the model has been identified from a converter which stray elements are perfectly defined, and no measurement errors are encountered. This contributes to the almost perfect agreement between the frequency model and the results obtained from the time domain simulation. However once again it should be reminded that the frequency model uses only linear equations in the frequency domain, whereas the time domain simulation is an actual representation of the switched behavior of the converter.
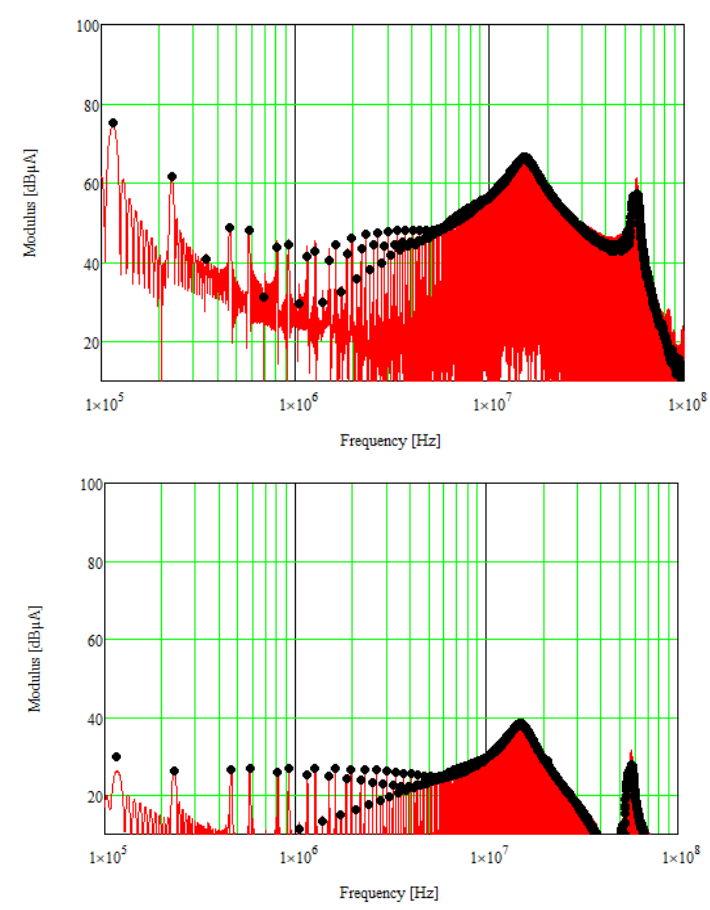

Fig. 9. Comparison between frequency model (black points) and time domain simulation + FFT (red) for minus to ground (Top $I_{1}$, Bottom $I_{2}$ )

\section{EXPERIMENTAL RESULTS}

The experimental validation is really mandatory when dealing with EMC aspects, since ElectroMagnetic Interferences (EMI) are not always circulating where expected, what leads any kind of modeling quite challenging. Therefore, the same identification process has been carried out using an experimental Boost converter. The experimental setup is displayed in Fig. 10. A specific interface has been built for impedance measurement, to provide measurement reproducibility. It is composed of several $\mathrm{BNC}$ connectors, to link the converter with the impedance measurement bridge, but also allowing to short circuit some parts of the converter. Using a BNC short circuit and a BNC cable to the impedance meter allows performing the three measurement configurations illustrated in Fig. 3, with good reproducibility. Obviously the impedance bridge is calibrated accounting for the BNC cable.

The second step of the model identification is then performed using a time domain recording with two high bandwidth current probes $(50 \mathrm{MHz})$ and high number of samples (500k). To keep information on phase shift, the two line currents are measured simultaneously. The identification is performed only on the relevant peaks of the FFT. Care has to be taken to the numerical processing of the signals to avoid a bad evaluation of the amplitude of the peaks: a mismatch in the exact frequency may result in a large error in the peak amplitude and therefore in the model evaluation. This point was not critic in simulation, since the switching frequency was perfectly known, but in measurement, a small jitter is always present and peak search becomes critical.

Once identified, the frequency model has been implemented in the same frequency solver as in section III. Results are compared to the measurement realized in the same test conditions (asymmetric LISN in Fig. 11 and minus to ground in Fig. 12). Even if not as "perfect" as in simulation, the overall results of Fig. 11 are good. The main differences 
are attributed to the model of the LISN, which is not exactly represented by the circuit of Fig. 5, due to its stray behavior. In Fig. 12, the high frequency difference is attributed to the impact of the impedance of the short circuit, which was estimated with a $5 \mathrm{nH}-265 \mathrm{~m} \Omega$ circuit, but with some possible errors. Also, the very low value of $\mathrm{I}_{2}$ in this configuration leads to very high inaccuracy. The low frequency mismatch between $500 \mathrm{kHz}$ and $3 \mathrm{MHz}$ is due to a disturbance of the converter operating point: the control circuit is directly fed from the power source, and short circuiting the LISN minus results in less voltage drop and therefore a small change in input voltage and thus duty cycle. However, the global matching between the model and the measurement is quite good, what validates the model, as well as the identification method.

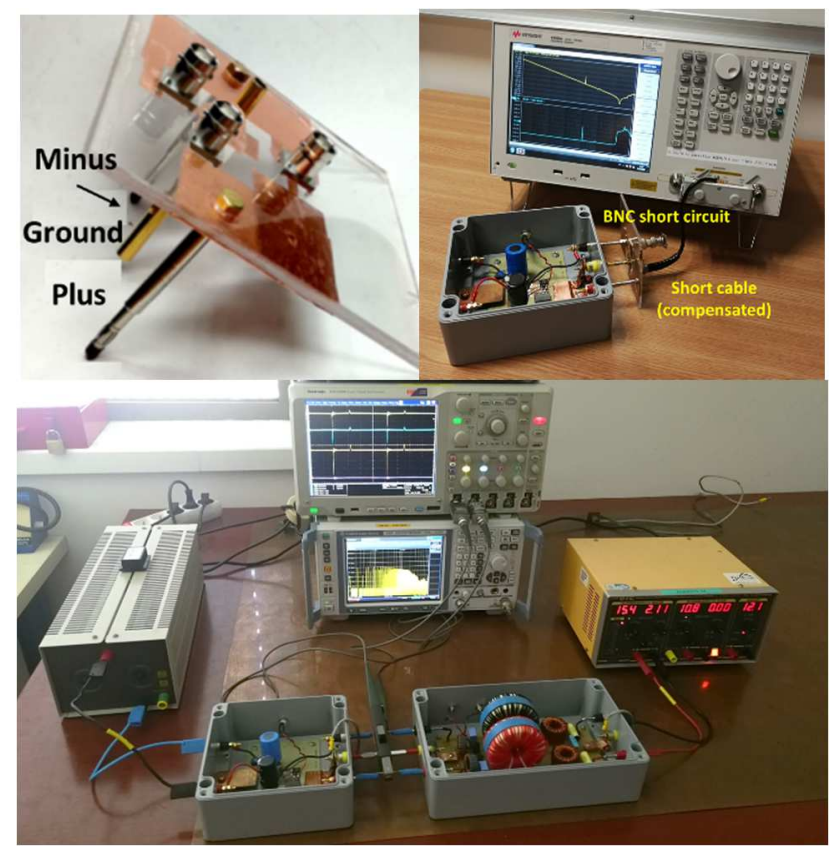

Fig. 10. Experimental setup. Top: impedance measurement with dedicated interface to connect the converter to the impedance bridge. Bottom, LISN and Boost converter during line current measurement in the time domain.
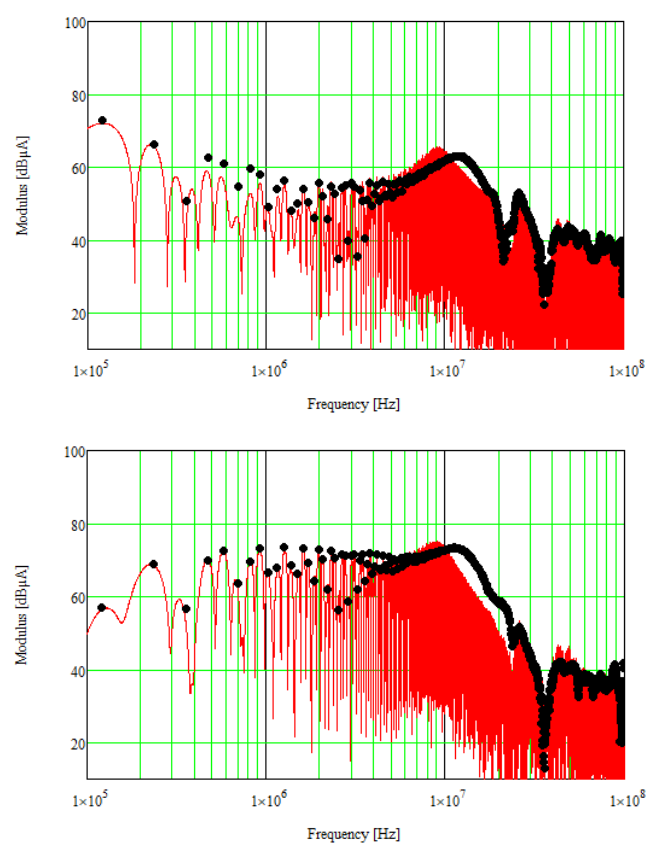

Fig. 11. Comparison between frequency model (black points) and measurement (red) for asymmetric LISN (Top $\mathrm{I}_{1}$, Bottom $\mathrm{I}_{2}$ )
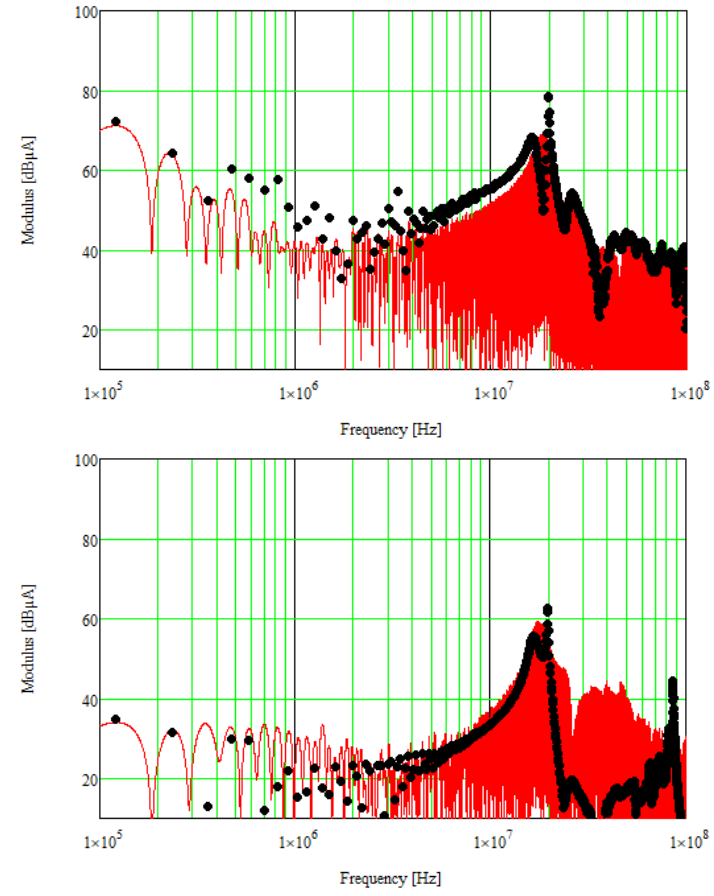

Fig. 12. Comparison between frequency model (black points) and measurement (red) for minus to ground (Top $\mathrm{I}_{1}$, Bottom $\mathrm{I}_{2}$ )

\section{DISCUSSION AND CONCLUSION}

\section{A. A Continuous Model to Represent Switching Devices?}

After having introduced the "Black Box" model as well as the identification method without any assumption on the model symmetry, it has been shown that the model reproduces quite well the EMC behavior of the converter, even in study cases different from the one used for identification. This shows that the switching behavior can be replaced by equivalent sources and impedances. To justify further this representation, an analysis has been performed on CM and DM impedance measurement of the Boost converter, using three different states for the switches. In the first one, the MOSFET is in the on-state and the diode in the off-state (replaced by a $10 \mathrm{pF}$ constant capacitance). In the second case, the MOSFET is off (replaced by Coss(Vds) from Pspice model) and the diode is on. The last state corresponds to the identification state: both devices are off, and replaced by their output capacitances. As noticed in Fig. 13, the difference in DM impedance is not noticeable, whereas it just adds a small resonance below $1 \mathrm{MHz}$ for $\mathrm{CM}$ Impedance. The impedances being almost identical whatever the semiconductors state, it explains why the Black Box model is quite accurate to represent the EMC behavior.

The effect of switching not being included in the impedances, which can be simply identified from the offline converter measurement, it is of course contained in the sources Vex and Iex. These sources include all frequency content of the converter switching behavior. Therefore, if the switching frequency or the duty cycle changes, the disturbance sources will be modified accordingly. Also if the switching speed grows, then the high frequency content of these sources will also increase.

\section{B. Symmetric or Assymetric Model ?}

When introducing the paper, the concern about symmetric model $\left(Z_{21}=Z_{22}\right)$ and associated identification method based on mode separation was raised. Indeed, if the model is 
symmetrical, only two impedances need to be identified. This can be done measuring DM and CM impedances. Using the mode separation, equations (1) and (2) can be easily used to determine Vex and Iex based on converter operation in a known environment. Therefore, the model identification is quite simple [2]. However, when connected to a non symmetrical grid, the CM generation does not only depends on Vex, and the DM not only on Iex. This mode coupling may generate some errors, which can be quantified in the studied examples. For this purpose, the symmetric model has been identified, with DM and CM impedance measurement, and based on CM and DM current measurement, injected in Eq (1) and (2). This symmetrical model has been inserted in the two test cases described in subsection II D. Only experimental measurements on the test case "minus to ground" are presented in this section, in comparison with the asymmetric model (Fig. 14).

The comparison between the two models shows a better matching with measurement for the asymmetric model, beyond $15 \mathrm{MHz}$. Below this frequency range, both models predict the EMC generation quite well. Therefore, it is matter of tradeoff between identification effort and frequency validity. The mode separation method used in [2] works quite well and is easy to set up, but may fail to predict the noise in the high frequency range if the grid is very asymmetrical. Fig. 15 shows that the difference becomes less important for the study case with asymmetric LISN.

\section{Conclusion}

This paper has shown that a "Black Box" model can be identified with a two-step method. The first step uses an offline impedance measurement to obtain the 3 different impedances. The second step uses the measurement of line currents in a known environment. This allows identifying the sources of the model, Vex and Iex. The identification process has been implemented and validated in simulation and in measurement, leading to a better accuracy in the high frequency range than the usual symmetric model, which can be identified a bit easier, using the mode separation assumption. The good results obtained in this paper, also validate the concept of "Black Box Model", representing switching devices with time invariant impedances and simple excitation sources.

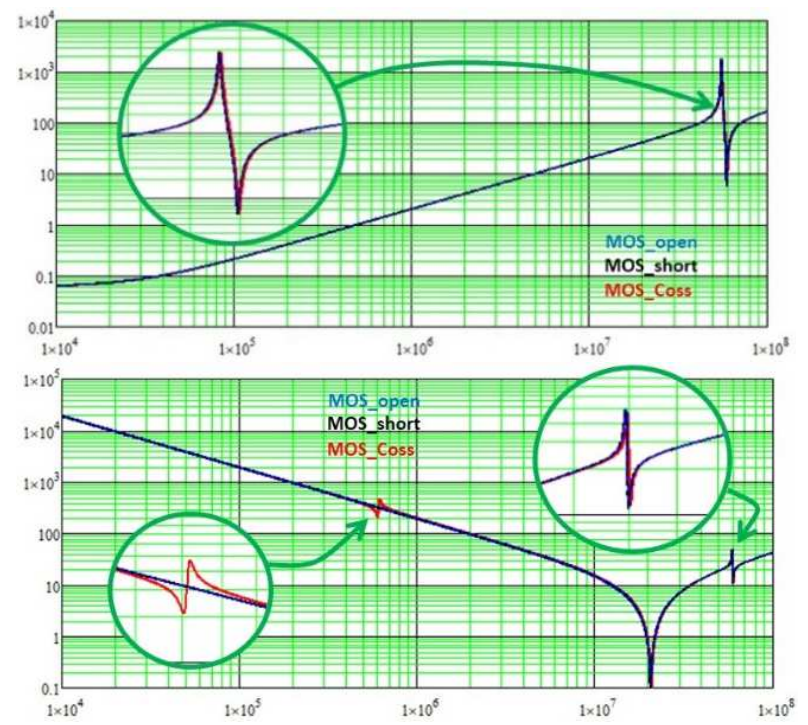

Fig. 13. Comparison of DM impedance (top) and CM impedance (bottom) for three representations of the switche states (AC sweep simulation using the circuit model of the converter from Fig. 7): MOS on, Diode off, MOS off, Diode on, and both devices replaced by their off state capacitors.
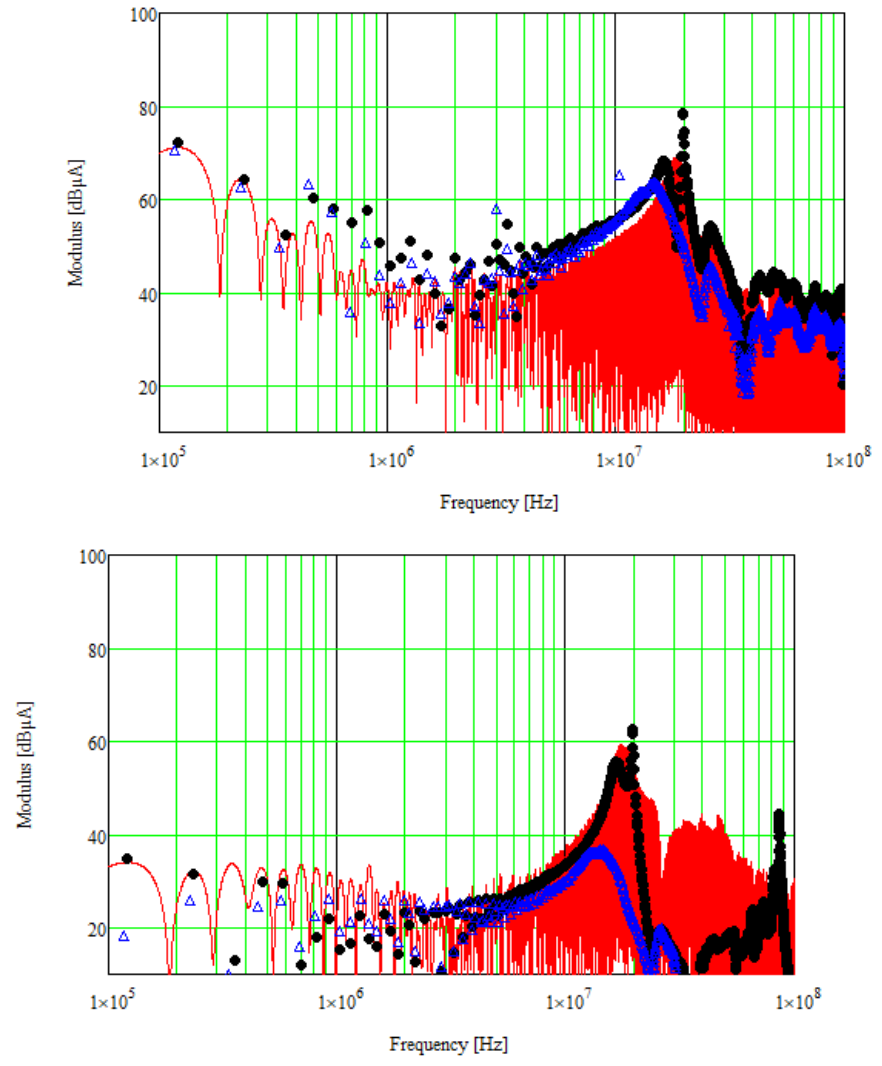

Fig. 14. Comparison between asymmetric frequency model (black points), symmetric frequency model (blue points) and measurement (red) for minus to ground test case (Top $\mathrm{I}_{1}$, Bottom $\mathrm{I}_{2}$ )
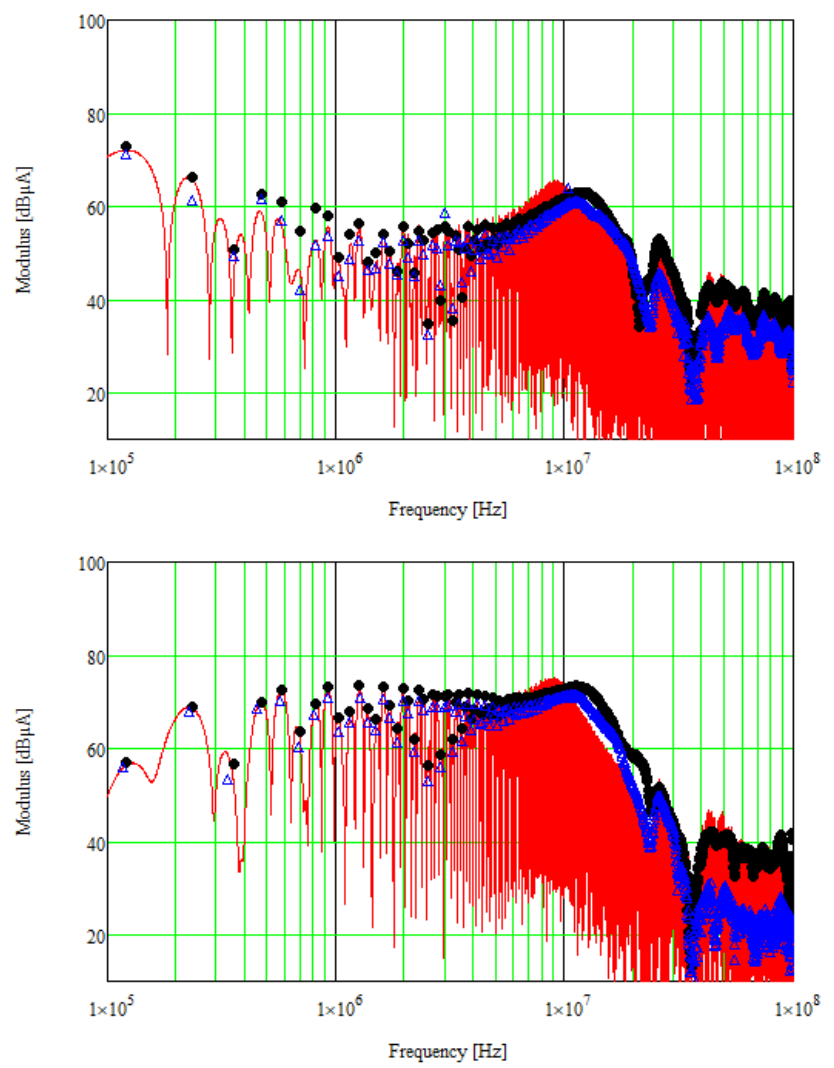

Fig. 15. Comparison between asymmetric frequency model (black points), symmetric frequency model (blue points) and measurement (red) for asymmetric LISN test case (Top $\mathrm{I}_{1}$, Bottom $\mathrm{I}_{2}$ ) 


\section{REFERENCES}

[1] B. Czerniewski, J. Schanen and P. Zanchetta, "EMC Generation and Propagation in Embedded Grids with Multiple Converters," PEDSTC 2019, Shiraz, Iran, pp. 433-438

[2] M. Amara, C. Vollaire, M. Ali and F. Costa, "Black Box EMC Modeling of a Three Phase Inverter," 2018 EMC EUROPE, Amsterdam, 2018, pp. 642-647

[3] H. Bishnoi, A. C. Baisden, P. Mattavelli and D. Boroyevich, "Analysis of EMI Terminal Modeling of Switched Power Converters," IEEE Trans Power Electronics, vol. 27, no. 9, Sept. 2012.

[4] M. Foissac, J. Schanen and C. Vollaire, "“Black box" EMC model for power electronics converter," IEEE Energy Conversion Congress and Exposition, San Jose, CA, 2009, pp. 3609-3615.

[5] B. Sun, R. Burgos and D. Boroyevich, "Common-Mode EMI Unterminated Behavioral Model of Wide-Bandgap-Based Power Converters Operating at High Switching Frequency," in IEEE Journal of Emerging and Selected Topics in Power Electronics, vol. 7, no. 4, Dec. 2019.
[6] A. Gahfif, P. É. Levy, M. Ali, M. Berkani and F. Costa, "EMC "Black Box" model for unbalanced power electronic converters," 2019 EMC EUROPE, Barcelona, Spain, pp. 957-962

[7] A. Ales, J. Schanen, D. Moussaoui and J. Roudet, "Impedances Identification of DC/DC Converters for Network EMC Analysis," in IEEE Transactions on Power Electronics, vol. 29, no. 12, pp. 64456457, Dec. 2014, doi: 10.1109/TPEL.2014.2302851.

[8] Ferran Martiacuten; Lei Zhu; Jiasheng Hong; Francisco Medina, "BALANCED POWER DIVIDERS/COMBINERS," in Balanced Microwave Filters , IEEE, 2018, pp.565-606, doi: 10.1002/9781119238386.ch15.

[9] https://fr.tek.com/datasheet/mixed-signal-oscilloscopes - online, accessed on July $20^{\text {th }}, 2021$ 